\title{
CMR survey in Thalassemia Intermedia patients
}

\author{
Antonella Meloni ${ }^{1 *}$, Maria Eliana Lai ${ }^{2}$, Stefania Vacquer ${ }^{2}$, Maddalena Lendini ${ }^{3}$, Petra Keilberg ${ }^{1}$, Maria Chiara Resta ${ }^{4}$, \\ Claudio Ascioti ${ }^{5}$, Vincenzo Positano ${ }^{1}$, Massimo Lombardi ${ }^{1}$, Alessia Pepe ${ }^{1}$ \\ From 17th Annual SCMR Scientific Sessions \\ New Orleans, LA, USA. 16-19 January 2014
}

\section{Background}

Little is known about cardiac involvement in thalassemia intermedia (TI) using cardiovascular magnetic resonance (CMR). We investigated myocardial iron overload (MIO), biventricular parameters, and myocardial fibrosis in a large cohort of TI patients, underlying the differences between transfusion-dependent and non-transfusiondependent patients.

\section{Methods}

We studied 252 adult TI patients (119 females, $39.5 \pm$ 10.4 years) enrolled in the MIOT Network. MIO was assessed using a multislice multiecho $\mathrm{T} 2 *$ approach. Biventricular function parameters were quantified by cine sequences. Myocardial fibrosis was evaluated by late gadolinium enhancement acquisitions.

\section{Results}

One-hundred and eighty-eight (74.6\%) patients showed no MIO in any segment, 56 (22\%) had an heterogeneous distribution ( 52 with global heart $\mathrm{T} 2 * \geq 20 \mathrm{~ms}$ ), and 8 (0.3\%) showed an homogeneous MIO. Left ventricular (LV) and right ventricular (RV) dilatations were present in $113(45 \%)$ and in $49(19 \%)$ patients, respectively. LV dysfunction was present in the $18.0 \%$ of the cases while RV dysfunction in the $3.63 \%$. High LV mass indexes were present in $22(8.7 \%)$ patients. Fifty-two/227 (22.9\%) patients showed myocardial fibrosis. Myocardial fibrosis was associated to LV dysfunction $(\mathrm{P}=0.001)$ and high mass indexes $(\mathrm{P}=0.038)$. One-hundred and fourteen patients were non-transfusion dependent (transfusion requirements absent or sporadic) while 138 patients were transfusion-dependent (regular transfusions). The mean age at start of chronic transfusions was $11.8 \pm$ 12.3 years. Table 1 shows the comparison between the two groups. Non-transfusion-dependent patients showed significantly higher global heart $\mathrm{T} 2$ * values and $\mathrm{MIO}$ with a global heart $\mathrm{T}^{*}<20 \mathrm{~ms}$ was detected in two of them (one requiring occasional blood transfusions and one non transfused). Biventricular end-diastolic volume index, stroke volume index, left ventricular (LV) mass index, and LV cardiac index were significantly higher in the non-transfusion dependent group.

\section{Conclusions}

CMR plays a key role in the management of TI patients. Heart iron (global heart T2* $<20 \mathrm{~ms}$ ) was not common, but a quarter of the patients had some pathological segments. A consistent number of patients had the stigmata of the high cardiac output state cardiomyopathy. Myocardial fibrosis was related to the high cardiac output state. The signs of the high output state were controlled in the transfusion-dependent-patients.

\section{Funding}

The MIOT project receives "no-profit support" from industrial sponsorships (Chiesi Farmaceutici S.p.A. and ApoPharma Inc.). This study was also supported by: "Ministero della Salute, fondi ex art. 12 D.Lgs. 502/92 e s.m.i., ricerca sanitaria finalizzata anno 2006" and "Fondazione L. Giambrone". 
Table 1

\begin{tabular}{|c|c|c|c|}
\hline & Non-transfusion-dependent & Transfusion-dependent & $P$ \\
\hline Age (years) & $39.9 \pm 11.5$ & $39.2 \pm 9.4$ & 0.922 \\
\hline $\operatorname{Sex}(M / F)$ & $67 / 47$ & $66 / 72$ & 0.083 \\
\hline Global heart T2* (ms) & $38.8 \pm 6.7$ & $35.5 \pm 9.2$ & 0.014 \\
\hline \multicolumn{4}{|l|}{ MIO pattern, $\mathrm{N}(\%)$ : } \\
\hline No MIO & $92(80.7)$ & $96(69.6)$ & 0.103 \\
\hline Heterogeneous MIO with global $\mathrm{T}^{*} \geq 20 \mathrm{~ms}$ & $20(17.5)$ & $32(23.2)$ & \\
\hline Heterogeneous MIO with global $\mathrm{T}^{*}<20 \mathrm{~ms}$ & $1(0.9)$ & $3(2.2)$ & \\
\hline Homogeneous MIO & $1(0.9)$ & $7(5.1)$ & \\
\hline LV end-diastolic volume index (ml/m2) & $99.4 \pm 19.6$ & $92.9 \pm 19.1$ & 0.009 \\
\hline LV end-systolic volume index (ml/m2) & $36.6 \pm 11.4$ & $34.9 \pm 10.4$ & 0.249 \\
\hline LV stroke volume index $(\mathrm{ml} / \mathrm{m} 2)$ & $62.9 \pm 12.4$ & $58.6 \pm 13.1$ & 0.007 \\
\hline LV mass index $(\mathrm{g} / \mathrm{m} 2)$ & $69.9 \pm 13.9$ & $63.9 \pm 12.9$ & 0.004 \\
\hline LV ejection fraction (\%) & $63.7 \pm 6.8$ & $62.5 \pm 6.6$ & 0.163 \\
\hline LV cardiac index (L/min/m2) & $7.6 \pm 2.3$ & $6.5 \pm 2.2$ & 0.002 \\
\hline LGE, N (\%) & 20/105 (19) & $32 / 122(26.2)$ & 0.199 \\
\hline RV end-diastolicvolume index (ml/m2) & $92.0 \pm 23.3$ & $86.5 \pm 20.8$ & 0.048 \\
\hline RV end-systolic volume index $(\mathrm{ml} / \mathrm{m} 2)$ & $32.7 \pm 14.9$ & $31.8 \pm 11.3$ & 0.571 \\
\hline RV stroke volume index (ml/m2) & $58.5 \pm 14.9$ & $54.5 \pm 14.3$ & 0.017 \\
\hline RV ejection fraction (\%) & $64.7 \pm 8.3$ & $63.3 \pm 7.5$ & 0.168 \\
\hline
\end{tabular}

$\mathrm{MIO}=$ myocardial iron overload; $\mathrm{LV}=$ left ventricular; RV = right ventricular

\section{Authors' details}

${ }^{1}$ CMR Unit, Fondazione G.Monasterio CNR-Regione Toscana and Institute of Clinical Physiology, Pisa, Italy. ${ }^{2}$ Ospedale microcitemico, Centro Talassemici Adulti, Cagliari, Italy. ${ }^{3}$ Centro trasfusionale, Osp. Giovanni Paolo II, Olbia, Italy. ${ }^{4}$ Struttura Complessa di Radiologia, OSP. SS. Annunziata ASL Taranto,

Taranto, Italy. ${ }^{5}$ Struttura Complessa di Cardioradiologia, P.O. "Giovanni Paolo

II", Lamezia Terme, Italy.

Published: 16 January 2014

\section{Submit your next manuscript to BioMed Central} and take full advantage of:

- Convenient online submission

- Thorough peer review

- No space constraints or color figure charges

- Immediate publication on acceptance

- Inclusion in PubMed, CAS, Scopus and Google Scholar

- Research which is freely available for redistribution

Submit your manuscript at www.biomedcentral.com/submit 